\title{
BILATERAL APPROXIMATIONS AND PERIODIC SOLUTIONS OF SYSTEMS OF DIFFERENTIAL EQUATIONS WITH IMPULSES
}

\author{
S.G. HRistova AND D.D. BAinov
}

\begin{abstract}
The paper justifies a method of bilateral approximations for finding the periodic solution of a non-linear system of differential equations with impulsive perturbations at fixed moments of time.
\end{abstract}

Systems with impulses find a growing application in control theory, radiotechnology, biology, and so on. The works of Millman and Mishkis [4], [5] mark the beginning of scientists' concern with these systems. Among the papers published after that we will mention [6]-[12]. However, the results obtained in these contributions are mainly of qualitative character. In the solution of a number of problems related to mathematical modelling of processes with the help of systems with impulses, it is necessary for various problems concerning these systems to be solved approximately with exactness given beforehand. Another reason to elaborate approximate analytic and numeric methods is the fact that their exact solution is possible only in extraordinary cases. An efficient approximate method is the one of bilateral approximations both from the theoretical and the practical viewpoint. It consists of constructing monotone sequences of functions that restrict the solution from above and from below. Thus an

Received 25 October 1984.

Copyright Clearance Centre, Inc. Serial-fee code: 0004-9727/85 $\$$ A2.00 + 0.00 . 
estimate of the error of the approximate solution is comparatively easily obtained.

The present paper justifies a variant of the method of bilateral approximations for finding an approximate periodic solution of systems of ordinary differential equations with impulses.

Note that the method of bilateral approximations for initial value and periodic problems concerning ordinary differential equations without impulses has been justified in [2] and [3].

Consider the system of differential equations with impulse perturbations at fixed moments of time

$$
\begin{aligned}
\dot{x} & =f(t, x, x) \text { for } t \neq t_{i}, \\
\left.\Delta x\right|_{t=t_{i}} & =I_{i}(x)
\end{aligned}
$$

where $x \in R^{n}, f: R \times D \times D \rightarrow R^{n}, I_{i}: D \rightarrow R^{n} \quad(i=0, \pm 1, \pm 2, \ldots)$. $t_{i} \in R \quad(i=0, \pm 1, \pm 2, \ldots)$ are fixed points, such that $t_{i+1}>t_{i}$ and

$$
\lim _{i \rightarrow \pm \infty} t_{i}= \pm \infty,\left.\Delta x\right|_{t=t_{i}}=x\left(t_{i}+0\right)-x\left(t_{i}-0\right), \quad D=\left\{x \in R^{n}: a \leq x \leq b\right\} \text {, }
$$

$a, b \in R^{n}$ are fixed.

The following notation will be used: $x \leq y$ if $x_{i} \leq y_{i}$, $i=1, \ldots, n$, where $x=\left(x_{1}, x_{2}, \ldots, x_{n}\right), y=\left(y_{1}, y_{2}, \ldots, y_{n}\right)$.

We will say that conditions $(A)$ are fulfilled if the following conditions hold:

(Al) the function $f(t, x, y): R \times D \times D \rightarrow R^{n}$ is continuous in all its arguments, it is periodic in $t$ with period $T$, it is non-decreasing in $x$, it is non-increasing in $y$ and satisfied the inequalities

$$
m \leq f(t, x, y) \leq M, x, y \in D,
$$

where $m, M \in R^{n}$;

(A2) a natural number $p$ exists such that 


$$
\begin{aligned}
I_{i+p}(x) & =I_{i}(x), \quad x \in D, \\
t_{i+p} & =t_{i}+T, \quad i=0, \pm 1, \pm 2, \ldots ;
\end{aligned}
$$

(A3) the functions $I_{i}: D \rightarrow R^{n}, i=0, \pm 1, \pm 2, \ldots$ are defined, continuous and non-decreasing in $D$ and they satisfy the inequality

$$
z_{i} \leq I_{i}(x) \leq L_{i}, \quad x \in D,
$$

where $z_{i}, L_{i} \in R^{n} \quad(i=0, \pm 1, \pm 2, \ldots), L_{i} \geq 0$,

$\imath_{i} \leq 0$

(A4) the inequality

$$
b-a \geq \frac{T}{2}(M-m)+2\left(\sum_{i=1}^{p} L_{i}-\sum_{i=1}^{p} I_{i}\right)
$$

holds.

We are looking for a periodic solution with period $T$ of system (1) which for $t=0$ passes through the point $x_{0} \in D_{B}$, where

$$
D_{\beta}=\left\{x \in R^{n}: a+\beta \leq x \leq b-\beta\right\}, \quad \beta=\frac{T}{4}(M-m)+\sum_{i=1}^{p}\left(L_{i}-z_{i}\right) \text {. }
$$

Define two sequences of functions $\left\{u_{n}(t)\right\}_{0}^{\infty}$ and $\left\{v_{n}(t)\right\}_{0}^{\infty}$, $t \in[0, T]$ with the help of the recurrence formulae

(2) $u_{n+1}(t)=x_{0}+\left(1-\frac{t}{T}\right) \int_{0}^{t} f\left(s, u_{n}(s), v_{n}(s)\right) d s$

$$
\begin{aligned}
& -\frac{t}{T} \int_{t}^{T} f\left(s, v_{n}(s), u_{n}(s)\right) d s \\
& +\left(1-\frac{t}{T}\right) \sum_{0<t_{i}<t} I_{i}\left(u_{n}\left(t_{i}\right)\right)-\frac{t}{T} \sum_{t<t_{i}<T} I_{i}\left(v_{n}\left(t_{i}\right)\right),
\end{aligned}
$$


(3) $v_{n+1}(t)=x_{0}+\left(1-\frac{t}{T}\right) \int_{0}^{t} f\left(s, v_{n}(s), u_{n}(s)\right) d s$

$$
\begin{gathered}
-\frac{t}{T} \int_{t}^{T} f\left(s, u_{n}(s), v_{n}(s)\right) d s \\
+\left(1-\frac{t}{T}\right) \sum_{0<t}<t I_{i}\left(v_{n}\left(t_{i}\right)\right)-\frac{t}{T} \sum_{t<t_{i}<T} I_{i}\left(u_{n}\left(t_{i}\right)\right), \\
u_{0}(t)=x_{0}-\alpha(t) \frac{M-m}{2}+\sum_{0<t i t} z_{i}-\frac{t}{T} \sum_{0<t_{i}<T} L_{i}, \\
v_{0}(t)=x_{0}+\alpha(t) \frac{M-m}{2}+\sum_{0<t_{i}<t} L_{i}-\frac{t}{T} \sum_{0<t_{i}<T} z_{i},
\end{gathered}
$$

where $\alpha(t)=2 t(1-(t / T))$.

LEMMA 1. Let conditions (A) hold and let $x_{0} \in D_{B}$.

Then the functions $u_{n}(t)$ and $v_{n}(t), n \geq 1$, satisfy the conditions

$$
\begin{aligned}
& u_{n}(t) \in D, \quad v_{n}(t) \in D, \\
& u_{n}(0)=u_{n}(T)=x_{0}, v_{n}(0)=v_{n}(T)=x_{0},
\end{aligned}
$$

and

$$
u_{0}(t) \leq u_{1}(t) \leq \ldots \leq u_{n}(t) \leq \ldots \leq v_{n}(t) \leq \ldots \leq v_{1}(t) \leq v_{0}(t) .
$$

Proof. Equality (5) is implied by the recurrence formulae (2) and (3).

Conditions (AI) and (A3) yield that for any $n \geq 0$ the inequalities

$$
\begin{aligned}
& u_{n+1}(t) \geq x_{0}-\frac{T}{4}(M-m)-\sum_{i=1}^{p}\left(L_{i}-\tau_{i}\right)=x_{0}-\beta, \\
& v_{n+1}(t) \geq x_{0}+\frac{T}{4}(M-m)+\sum_{i=1}^{p}\left(L_{i}-\tau_{i}\right)=x_{0}+\beta,
\end{aligned}
$$

hold.

We will prove that for $t \in[0, T]$ the inequalities 


$$
u_{n}(t) \leq v_{n}(t), n \geq 0 \text {, }
$$

hold.

Indeed, for $n=0$, the definition of the functions $u_{0}(t)$ and $v_{0}(t)$ implies that

$$
u_{0}(t) \leq v_{0}(t) \text { for } t \in[0, T]
$$

Conditions (Al) and (A3) and equalities (2) and (3) yield that

$$
u_{1}(t) \leq v_{1}(t), \quad t \in[0, T] \text {. }
$$

Applying the method of mathematical induction we obtain that inequality (9) is satisfied for every $n \geq 0$. Inequalities (7), ( 8 ) and (9) imply that for $n \geq 0$ the relations $u_{n}(t) \in D, v_{n}(t) \in D$ hold.

We will prove that inequalities (6) are satisfied. Conditions (Al) and (A3) and equalities (2) and (3) yield that

$$
\begin{aligned}
& u_{1}(t) \geq x_{0}-\alpha(t) \frac{M-m}{2}+\sum_{0<t_{i}<t} z_{i}-\frac{t}{T} \sum_{0<t_{i}<T} L_{i}=u_{0}(t), \\
& v_{1}(t) \leq x_{0}+\alpha(t) \frac{M-m}{2}+\sum_{0<t_{i}<t} L_{i}-\frac{t}{T} \sum_{0<t_{i}<T} z_{i}=v_{0}(t) .
\end{aligned}
$$

Then in view of conditions (Al) and (A3) we get

$$
\begin{aligned}
u_{2}(t) \geq x_{0}+(1 & \left.-\frac{t}{T}\right) \int_{0}^{t} f\left(s, u_{0}(s), v_{0}(s)\right) d s-\frac{t}{T} \int_{t}^{T} f\left(s, v_{0}(s), u_{0}(s)\right) d s \\
& +\left(1-\frac{t}{T}\right) \sum_{0<t_{i}<t} I_{i}\left(u_{0}\left(t_{i}\right)\right)-\frac{t}{T} \sum_{t<t_{i}<T} I_{i}\left(v_{0}\left(t_{i}\right)\right)=u_{1}(t) .
\end{aligned}
$$

Analogously we obtain that $v_{2}(t) \leq v_{1}(t)$. By induction it is verified that the inequalities

$$
\begin{aligned}
& u_{0}(t) \leq u_{1}(t) \leq \ldots \leq u_{n}(t) \leq \ldots, \\
& v_{0}(t) \geq v_{1}(t) \geq \ldots \geq v_{n}(t) \geq \ldots,
\end{aligned}
$$

hold.

Inequalities (9) and (10) imply inequality (6). 
Thus Lemma 1 is proved.

LEMMA 2. Let the conditions (A) hold and let $x_{0} \in D_{B}$.

Then the sequences of functions $\left\{u_{n}(t)\right\}_{0}^{\infty}$ and $\left\{v_{n}(t)\right\}_{0}^{\infty}$ are convergent in the interval $[0, T]$ and their limits $U(t)=\lim _{n \rightarrow \infty} u_{n}(t)$, $V(t)=\lim _{n \rightarrow \infty} v_{n}(t)$ are piecewise continuous functions for which the conditions

$$
\begin{aligned}
& U(t) \in D, \quad V(t) \in D \text { for } t \in[0, T], \\
& U(t) \leq V(t) \text { for } t \in[0, T], \\
& U(0)=U(T)=x_{0}, \quad V(0)=V(T)=x_{0},
\end{aligned}
$$

are satisfied, and

$$
\begin{aligned}
U(t)=x_{0}+\left(1-\frac{t}{T}\right) & \int_{0}^{t} f(s, U(s), V(s)) d s-\frac{t}{T} \int_{t}^{T} f(s, V(s), U(s)) d s \\
& +\left(1-\frac{t}{T}\right) \sum_{0<t_{i}<t} I_{i}\left(U\left(t_{i}\right)\right)-\frac{t}{T} \sum_{t<t_{i}<T} I_{i}\left(V\left(t_{i}\right)\right), \\
V(t)=x_{0}+\left(1-\frac{t}{T}\right) \int_{0}^{t} f(s, V(s), U(s)) d s-\frac{t}{T} \int_{t}^{T} f(s, U(s), V(s)) d s & +\left(1-\frac{t}{T}\right) \sum_{0<t_{i}<t} I_{i}\left(V\left(t_{i}\right)\right)-\frac{t}{T} \sum_{t<t_{i}<T} I_{i}\left(U\left(t_{i}\right)\right) .
\end{aligned}
$$

Proof. Let $t_{i} \in(0, T), i=1, \ldots, p$. Consider the interval $\left[0, t_{1}\right]$. For $t \in\left[0, t_{1}\right\}$, the sequences of functions $\left\{u_{n}(t)\right\}_{0}^{\infty}$ and $\left\{v_{n}(t)\right\}_{0}^{\infty}$ are uniformly bounded, they are equicontinuous and hence there exist uniformly convergent subsequences $\left\{u_{n}^{(0)}(t)\right\}_{0}^{\infty}$ and $\left\{v_{n}^{(0)}(t)\right\}_{0}^{\infty}$.

$$
\begin{aligned}
& u^{(0)}(t)=\lim _{n \rightarrow \infty} u_{n}^{(0)}(t) \text { for } t \in\left[0, t_{1}\right], \\
& v^{(0)}(t)=\lim _{n \rightarrow \infty} v_{n}^{(0)}(t) .
\end{aligned}
$$


Consider the interval $\left(t_{1}, t_{2}\right)$ and the sequences of functions $\left\{u_{n}^{(0)}(t)\right\}_{0}^{\infty}$ and $\left\{v_{n}(t)\right\}_{0}^{\infty}$, defined and continuous in this interval. Besides the values of the functions $u_{n}^{(0)}(t)$ and $v_{n}^{(0)}(t)$ at the point $t=t_{1}$ we have $u_{n}^{(0)}\left(t_{1}+0\right)=u_{n}^{(0)}\left(t_{1}\right)+I_{1}\left(u_{n}^{(0)}\left(t_{1}\right)\right)$ and $v_{n}^{(0)}\left(t_{1}+0\right)=v_{n}^{(0)}\left(t_{1}\right)+I_{1}\left(v_{n}^{(0)}\left(t_{1}\right)\right)$, respectively.

For $t \in\left(t_{1}, t_{2}\right]$, the sequences of functions are uniformly bounded and equicontinuous and hence there exist convergent subsequences $\left\{u_{n}^{(1)}(t)\right\}_{0}^{\infty}$ and $\left\{v_{n}^{(1)}(t)\right\}_{0}^{\infty}$. Introduce the notation:

$$
\begin{aligned}
& u^{(1)}(t)=\lim _{n \rightarrow \infty} u_{n}^{(1)}(t), \\
& \text { for } t \in\left[t_{1}, t_{2}\right] \text {. }
\end{aligned}
$$

$$
v^{(1)}(t)=\lim _{n \rightarrow \infty} v_{n}^{(1)}(t)
$$

The equalities

$$
u^{(1)}\left(t_{1}\right)=u^{(0)}\left(t_{1}\right)+I_{1}\left(u^{(0)}\left(t_{1}\right)\right)
$$

and

$$
v^{(1)}\left(t_{1}\right)=v^{(0)}\left(t_{1}\right)+I_{1}\left(v^{(0)}\left(t_{1}\right)\right)
$$

hold.

Analogously we define functions $u^{(k)}(t)$ and $v^{(k)}(t)$ for $t \in\left[t_{k}, t_{k+1}\right]$ or $t \in[t \rho, T\rfloor$. Consider the functions $U(t)$ and $V(t)$ defined for $t \in[0, T]$ by the equalities

$$
u(t)= \begin{cases}u^{(0)}(t) \text { for } t \in\left[0, t_{1}\right], \\ u^{(k)}(t) \text { for } t \in\left(t_{k}, t_{k+1}\right], k=1, \ldots, \rho-1, \\ u^{(\rho)}(t) \text { for } t \in(t \rho, T],\end{cases}
$$




$$
V(t)= \begin{cases}v^{(0)}(t) & \text { for } t \in\left[0, t_{1}\right], \\ v^{(k)}(t) & \text { for } t \in\left(t_{k}, t_{k+1}\right], k=1, \ldots, \rho-1, \\ v^{(\rho)}(t) & \text { for } t \in(t \rho, T] .\end{cases}
$$

The functions $U(t)$ and $V(t)$ are piecewise continuous with first order discontinuities at the points $t_{i}, i=1, \ldots, p$, and satisfy the jump condition

$$
U\left(t_{i}+0\right)-U\left(t_{i}-0\right)=I_{i}\left(U\left(t_{i}-0\right)\right)
$$

and

$$
V\left(t_{i}+0\right)-V\left(t_{i}-0\right)=I_{i}\left(V\left(t_{i}-0\right)\right)
$$

Inequalities (6) imply that for $k=0,1, \ldots, \rho$, the inequalities $u^{(k)}(t) \leq v^{(k)}(t)$ are satisfied, for $t \in\left(t_{k}, t_{k+1}\right]$, and hence

$$
U(t) \leq V(t), \quad t \in[0, T] .
$$

Equality $(5)$ yields that $u^{(0)}(0)=u^{(\rho)}(T)$ and $v^{(0)}(0)=v^{(\rho)}(T)$ and hence

$$
\begin{aligned}
& U(0)=U(T)=x_{0}, \\
& V(0)=V(T)=x_{0} .
\end{aligned}
$$

Since the set $D$ is closed, it follows that $U(t) \in D, V(t) \in D$, $t \in[0, T]$. Taking limits in equalities (2) and (3) we obtain that the functions $U(t)$ and $V(t)$ satisfy system (11).

Thus Lemma 2 is proved.

Consider the system

$$
\begin{aligned}
x(t)=x_{0}+\left(1-\frac{t}{T}\right) & \int_{0}^{t} f(s, x(s), x(s)) d s-\frac{t}{T} \int_{t}^{T} f(s, x(s), x(s)) d s \\
& +\left(1-\frac{t}{T}\right) \sum_{0<t_{i}<t} I_{i}\left(x\left(t_{i}\right)\right)-\frac{t}{T} \sum_{t<t_{i}<T} I_{i}\left(x\left(t_{i}\right)\right) .
\end{aligned}
$$

LEMMA 3. Let the following conditions hold: 
(1) conditions (A) are fulfilled and $x_{0} \in D_{B}$;

(2) the functions $I_{i}(x), i=1, \ldots, \rho$, satisfy in the set $D$ the condition $I_{i}(x)-I_{i}(y) \leq Q_{i}(x-y)$, for $x \geq y$, where $Q_{i}, i=1, \ldots, \rho$ are $n \times n$ matrices with nonnegative elements;

(3) the inequality $2\left\|\sum_{i=1}^{\rho} Q_{i}\right\|<1$ holds.

Then the system (12) has at least one solution $x^{*}(t) \in D$, for which $x^{*}(0)=x^{*}(T)=x_{0}$.

Proof. Consider the set $\Omega$ consisting of all piecewise continuous functions $x(t)$ with discontinuities at the points $t_{i}, i=1, \ldots, p$, defined in the interval $[0, T]$ and belongong to the domain $D$ for $t \in[0, T]$, and for which $x(0)=x(T)=x_{0}$.

Define the operator $F: \Omega \rightarrow \Omega, F=A_{1}+A_{2}$, where the operators $A_{i}, i=1,2$, are defined by the equalities

$$
\begin{aligned}
& A_{1} x(t)=x_{0}+\left(1-\frac{t}{T}\right) \int_{0}^{t} f(s, x(s), x(s)) d s-\frac{t}{T} \int_{t}^{T} f(s, x(s), x(s)) d s, \\
& A_{2} x(t)=\left(1-\frac{t}{T}\right) \sum_{0<t_{i}<t} I_{i}\left(x\left(t_{i}\right)\right)-\frac{t}{T} \sum_{t<t_{i}<T} I_{i}\left(x\left(t_{i}\right)\right) .
\end{aligned}
$$

The set $\Omega$ is bounded, closed and convex and the operator $F$ transforms it onto itself.

We will prove that the operator $A_{1}$ is compact in $\Omega$.

Let the sequence $x_{n}(t) \in \Omega$ be uniformly convergent to the function $x(t) \in \Omega$ for $t \in[0, T]$. The continuity of the function $f(t, x, y)$ implies that

$$
f\left(t, x_{n}(t), x_{n}(t)\right) \underset{n \rightarrow \infty}{\longrightarrow} f(t, x(t), x(t))
$$

uniformly for $t \in[0, T]$. 
Then, for $t \in[0, T], A_{1} x_{n} \underset{n \rightarrow \infty}{\longrightarrow} A_{1} x$ holds, that is, the operator $A_{1}$ is continuous on $\Omega$.

Also, for $x \in \Omega$ and $t \in[0, T]$, the inequalities

$$
\begin{aligned}
\left\|A_{1} x(t)\right\| & \leq\left\|x_{0}\right\|+\left\|\int_{0}^{t} f(s, x(s), x(s)) d s\right\|+\left\|\int_{0}^{T} f(s, x(s), x(s)) d s\right\| \\
& \leq\left\|x_{0}\right\|+2 M_{0} T
\end{aligned}
$$

hold, where $M_{\mathrm{O}}=\max (\|M\|,\|m\|)$.

If $\xi_{1}$ and $\xi_{2}$ are two arbitrary points from the interval $[0, T]$, then

$$
\begin{aligned}
\left\|A_{1} x\left(\xi_{1}\right)-A_{2} x\left(\xi_{2}\right)\right\| \leq\left\|\int_{\xi_{1}}^{\xi_{2}} f(s, x(s), x(s)) d s\right\| & \\
& +\left\|\frac{\xi_{1}-\xi_{2}}{T} \int_{0}^{T} f(s, x(s), x(s)) d s\right\| \leq 2 M_{0}\left\|\xi_{1}-\xi_{2}\right\| .
\end{aligned}
$$

In view of Arzella's theorem, the operator $A_{1}$ is compact.

Let $x_{1}(t), x_{2}(t) \in \Omega$. Then

$$
\left\|A_{2} x_{1}-A_{2} x_{2}\right\| \leq 2\left\|x_{1}(t)-x_{2}(t)\right\|_{0} \cdot\left\|\sum_{i=1}^{p} Q_{i}\right\|,
$$

where $\|\tau(t)\|_{0}=\max _{[0, T]}\|\tau(t)\|$.

Hence the operator $A_{2}$ is a contracting one. Then the operator $F x=x$ has at least one solution $x^{\star}(t) \in \Omega$.

Lemma 3 is thus proved.

LEMMA 4. Let the conditions of Lemma 3 be fulfizled.

Then the systein (12) has a solution $x^{*}(t) \in \Omega$ for which the inequalities

$$
U(t) \leq x^{*}(t) \leq V(t), \quad t \in[0, T],
$$

hold. 
Proof. In view of Lemma 3, equation (12) has at least one solution $x^{*}(t) \in \Omega$. Conditions (Al) and (A3) yield that the inequality

$$
x^{*}(t) \geq \dot{x_{0}}+\left(1-\frac{t}{T}\right) t m-\frac{t}{T}(T-t) M+\left(1-\frac{t}{T}\right) \sum_{0<t_{i}<t} z_{i}-\frac{t}{T} \sum_{i=1}^{p} L_{i} \geq u_{0}(t)
$$

holds.

Analogously we have that $x^{*}(t) \leq v_{0}(t)$. By induction we get that for every $n \geq 0$,

$$
u_{n}(t) \leq x^{*}(t) \leq v_{n}(t)
$$

is satisfied for $t \in[0, T]$.

Taking limits in (14) proves inequalities (13).

Lemma 4 is proved.

LEMMA 5. Let the following conditions be satisfied:

(1) the conditions of Lemma 3 hold;

(2) the function $f(t, x, y)$ satisfies the condition

$$
f(t, x, y)-f(t, x, y) \leq K_{1}(x-\bar{x})+K_{2}(y-y)
$$

in the domain $R \times D \times D$ for $x \geq \bar{x}$ and $y \leq \bar{y}$, where $K_{1}$ and $K_{2}$ are $n \times n$ matrices with non-negative elements;

(3) the eigenvalues of the matrix

$$
\left(K_{1}+K_{2}\right)\left(\frac{T}{3}+\frac{4 T}{3} \sum_{i=1}^{p} Q_{i}\right)+2 \sum_{i=1}^{p} Q_{i}
$$

are smaller than 1 in modulus.

Then the system (12) has a unique solution $x(t) \in \Omega, t \in[0, T]$.

Proof. Estimate the difference $v_{n}(t)-u_{n}(t), t \in[0, T]$. For $n=0$ we have 
304

S.G. Hristova and D.D. Bainov

$$
\begin{aligned}
v_{0}(t)-u_{0}(t) \leq 4 t\left(1-\frac{t}{T}\right)(M-m)+2 \sum_{i=1}^{p}\left(L_{i}-z_{i}\right) & \\
& \leq T(M-m)+2 \sum_{i=1}^{p}\left(L_{i}-z_{i}\right) \equiv M_{1} .
\end{aligned}
$$

For $n=1$ we obtain

$$
\begin{aligned}
& v_{1}(t)-u_{1}(t) \\
& \leq\left(1-\frac{t}{T}\right) \int_{0}^{t}\left(K_{1}+K_{2}\right)\left(v_{0}(s)-u_{0}(s)\right) d s+\frac{t}{T}\left(K_{1}+K_{2}\right) \int_{t}^{T}\left(v_{0}(s)-u_{0}(s)\right) d s \\
& \quad+\left(1-\frac{t}{T}\right) \sum_{0<t_{i}<t} Q_{i}\left(v_{0}\left(t_{i}\right)-u_{0}\left(t_{i}\right)\right)+\frac{t}{T} \sum_{t<t_{i}<T} Q_{i}\left(v_{0}\left(t_{i}\right)-u_{0}\left(t_{i}\right)\right) \\
& \leq 4\left(K_{1}+K_{2}\right)\left\{(M-m) \frac{T}{3}+\sum_{i=1}^{p}\left(L_{i}-L_{i}\right)\right\} t\left(1-\frac{t}{T}\right)+2 M_{1} \sum_{i=1}^{p} Q_{i} \\
& \leq N_{1} T+2 M_{1} \sum_{i=1}^{p} Q_{i} \equiv M_{2},
\end{aligned}
$$

where $N_{1}=\left(K_{1}+K_{2}\right)\left\{(M-m) \frac{T}{3}+\sum_{i=1}^{p}\left(L_{i}-z_{i}\right)\right\}$.

By induction we get that for every $n=2,3, \ldots$ the estimate

$$
v_{n}(t)-u_{n}(t) \leq 4 t\left(1-\frac{t}{T}\right) N_{n}+2 M_{n} \sum_{i=1}^{p} Q_{i}
$$

holds, where

$$
N_{n}=\left(K_{1}+K_{2}\right)\left(\frac{T}{3} N_{n-1}+2 M_{n-1} \sum_{i=1}^{p} Q_{i}\right) \text {, }
$$

$$
M_{n}=N_{n-1} T+2 M_{n-1} \sum_{i=1}^{p} Q_{i} .
$$

The necessary and sufficient condition for all solutions of the system of difference equations (15) to approach zero is that the eigenvalues of the matrix

https://doi.org/10.1017/S0004972700004767 Published online by Cambridge University Press 


$$
\left(\begin{array}{cc}
\left(K_{1}+K_{2}\right) \frac{T}{3} & 2 \sum_{i=1}^{p} Q_{i}\left(K_{1}+K_{2}\right) \\
T & 2 \sum_{i=1}^{p} Q_{i}
\end{array}\right)
$$

are smaller than 1 in modulus, that is in view of [1], p. 190,

$$
-1+\left(K_{1}+K_{2}\right) \frac{T}{3}+2 \sum_{i=1}^{p} Q_{i}<-\frac{4 T}{3}\left(K_{1}+K_{2}\right) \sum_{i=1}^{p} Q_{i}<1
$$

should hold.

Condition (3) of Lemma 5 yields that inequality (16) is satisfied and hence $v_{n}(t)-u_{n}(t) \rightrightarrows 0$ for $n \rightarrow \infty$ and $t \in[0, T]$, that is, $U(t)=V(t), \quad t \in[0, T]$. In view of Lemma 4 ,

$$
U(t)=V(t)=x^{*}(t) \text { for } t \in[0, T],
$$

where $x^{*}(t) \in \Omega$ is a solution of system (12).

Thus Lemma 5 is proved.

THEOREM 1. Let the conditions of Lenma 5 be satisfied.

Then system (1) has a T-periodic solution $x=\varphi(t)$ for which $\varphi(0)=x_{0}$ and $\varphi(t)=\lim _{n \rightarrow \infty} u_{n}(t)=\lim _{n \rightarrow \infty} v_{n}(t)$ for $t \in[0, T]$.

Proof. In view of Lemma 5 system (12) has a unique solution $\varphi(t)$ for $t \in[0, T]$, for which the equalities

$$
\varphi(0)=\varphi(T)=x_{0}
$$

and

$$
\varphi(t)=\lim _{n \rightarrow \infty} u_{n}(t)=\lim _{n \rightarrow \infty} v_{n}(t)=U(t)=V(t)
$$

hold.

Moreover, the function $\varphi(t)$ satisfies the system (1) for $t \in[0, T]$.

Thus Theorem 1 is proved. 


\section{References}

[1] V.P. Demidovich, Lectures on the mathematical theory of stability (Publishing House "Nauka", Moscow, 1976).

[2] N.S. Kurpel, I.N. Maiboloda, "On one way of constructing bilateral approximations to the solution of equations" (Russian), Ukrain. Mat. そ. 28 (1976), 602-610.

[3] N.S. Kurpel, V.I. Tivonchuk, "On a bilateral method for approximate solution of the Cauchy problem for ordinary differential equations" (Russian), Ukrain. Mat. Z. 27 (1975), 528-534.

[4] V.D. Millman, A.D. Mishkis, "On the stability of motion in the presence of impulses" (Russian), Sibirsk. Mat. Ž. 1 (1960), 233-237.

[5] V.D. Millman, A.D. Mishkis, "Random impulses in linear dynamic systems", Approximate methods for the solutzon of differential equations, (Russian), 64-81 (Publishing House of the Academic of Science, Ukrain. SSR, Kiev, 1963).

[6] S.G. Pandit, "On the stability of impulsively perturbed differential systems", BulZ. Austral. Math. Soc. 17 (1977), 423-432.

[7] S.G. Pandit, "Differential systems with impulsive perturbations", Pacific J. Math. 86 (1980), 553-560.

[8] S.G. Pandit, "Systems described by differential equations containing impulses: existence and uniqueness", Rev. Roumaine Math. Pures App 2. 26 (1981), 879-887.

[9] T. Pavlidis, "Stability of systems described by differential equations containing impulses", IEEE Trans. Automat. Control 12 (1967), 43-45.

[10] V. Raghavendra and M. Kama Monana Rao, "On stability of differential systems with respect to impulsive perturbations", J. Math. Anal. App Z. 48 (1974), 515-526.

[11] M. Rama Mohana Rao and V. Sree Hari Rao, "Stability of impulsively perturbed systems", Bull. Austral. Math. Soc. 16 (1977), 99-110. 
[12] V. Sree Hari Rao, "On boundedness of impulsively perturbed systems", Bulz. Austral. Math. Soc. 18 (1978), 237-242.

23 Oborishte Str.,

1504 Sofia,

Bulgaria. 